\title{
Potential feedback control for the power control in wireless sensor networks
}

\author{
Dat Duong Phan ${ }^{\mathrm{a}}$, Emmanuel Moulay ${ }^{\mathrm{b}}$, Patrick Coirault ${ }^{\mathrm{a}}$, Anne-Marie \\ Poussard $^{\mathrm{b}}$, Rodolphe Vauzelle ${ }^{\mathrm{b}}$ \\ ${ }^{a}$ LIAS (EA 6315), École Nationale Supérieure d'Ingénieurs de Poitiers, 2 rue Pierre \\ Brousse, TSA 41105, 86073 Poitiers Cedex 9, France \\ ${ }^{b}$ XLIM-SIC (UMR CNRS 7252), Université de Poitiers, 11 bd Marie et Pierre Curie, \\ BP 30179, 86962 Futuroscope Chasseneuil Cedex, France
}

\begin{abstract}
The aim of this article is to develop a new feedback stabilization strategy involving a nonlinear Potential Feedback Control (PFC) for scalar discrete-time systems with disturbances by using Lyapunov functions. The main interest of the PFC is to achieve the stabilization with a constraint on the state space. We apply this strategy for the uplink power control in a real Wireless Sensor Network (WSN) and compare with the Adaptive Transmission Power Control (ATPC) usually used in telecommunications.
\end{abstract}

Keywords: Potential feedback control, discrete-time systems, Lyapunov functions, wireless sensor networks

Email addresses: dat.duong.phan@univ-poitiers.fr (Dat Duong Phan), emmanuel.moulay@univ-poitiers.fr (Emmanuel Moulay), patrick.coirault@univ-poitiers.fr (Patrick Coirault), anne.marie.poussard@univ-poitiers.fr (Anne-Marie Poussard), rodolphe.vauzelle@univ-poitiers.fr (Rodolphe Vauzelle) 


\section{Introduction}

Applications of WSN have dramatically increased in recent years, spanning environment monitoring, medical systems, smart building, nuclear industry and robotic. Such networks are composed of distributed nodes. Each node has sensor functionality, embedded processor and radio communication. For the star network topology, these nodes use only a single hope to send the monitoring data to a Central Server (CS) that is connected to the wired infrastructure. A WSN is usually composed of low-power operated battery devices and it is not possible in general to replace batteries due to the number of nodes deployed or the difficult access conditions. So, the battery conservation is a critical issue to prolong the network lifetime. One of the source of energy consumption is the radio transmission. In order to reduce the energy consumption, the idea is to design a power control for each node. Nevertheless, a low power transmission may cause errors in transmitted packets that are to be discarded, and the follow-on retransmission increases energy consumption. On the other side, a high power transmission achieves a good data transmission but consumes energy. So, a good power transmission control leads to a trade-off between the energy consumption and a low bit error rate $[1]$.

In this article, we focus on the uplink power control with the ZigBee standard [2]. Moreover, we suppose that the WSN has a star network topology with a CS performing calculations and several wireless sensors (WS). So, we do not consider routing between the WSs. With the star network topology, 
the CS and all WSs are fixed. In general, the constructor of WSNs sets the transmission power of each WS at the maximum power $[3,4]$. The fact that the power transmission is always at the maximum power for any link between the CS and the WS motivates us to develop a controller allowing to reduce the transmission power by keeping a good link's quality. To achieve the uplink power control in WSNs, we use an indicator of the transmission quality called the Received Signal Strength Indication (RSSI). The power control must achieve two main goals:

- a good Quality of Service (QoS) in WSNs by ensuring that the RSSI stays above a minimum RSSI, denoted $\mathrm{RSSI}_{\text {min }}$;

- a minimization of the energetic consumption. This allows to increase the autonomy of devices.

Both objectives mean to have a RSSI just above the $\mathrm{RSSI}_{\text {min }}$.

The development of WSNs in recent years has motivated the investigation of several power control strategies. The ATPC algorithm is proposed in [5]. Another approach which uses the theory of S-modular games is developed in [6]. A feedback control strategy is studied in [7] where the authors developed a robust distributed active power control by using a quantitative feedback theory approach. The main problem of the feedback control strategy for the power control of a WSN is due to the fact that there is a constraint on the RSSI which is the state of the system. It is possible to develop a Model Predictive Control (MPC) approach for a discrete-time system taking into 
account a constraint on the state variables $[8,9]$.

In our article, we provide a new simple Potential Feedback Control (PFC) strategy for a scalar system having a constraint on its state variable. It is an original approach, first developed in [10] for the power control in LTE, where the stabilization problem of a linear scalar system with a constraint on the state space is transformed into a problem of nonlinear stabilization. The PFC has essentially been used in robotic to avoid the collisions with obstacles $[11,12]$. It is a nonlinear control whose nonlinear part is a virtual Coulomb potential barrier ensuring that the state space cannot cross this barrier. Moreover, the complexity of the proposed control algorithm is quite low because we can easily tune outline one gain for each wireless sensor. This is a good feature for our real application. The main difficulty with the potential control is due to the nonlinearity of the potential term which implies the use of the Lyapunov theory. In this article, we extend the validity of the PFC developed in [10] and we apply this strategy to a real WSN platform. Moreover, we compare the PFC strategy with the ATPC and MPC strategies.

The paper is organized as follows. The ATPC for WSNs is presented in the section 2. A PFC for scalar discrete-time systems with disturbances is built in the section 3. Then, the PFC strategy is applied to a real WSN composed of four WSs and compared with the ATPC and MPC strategy in the section 4. The energy saving result coming from the power control is also presented in the section 4. Finally, a conclusion is addressed in the section 5. 


\section{Implementation of the ATPC strategy}

In this subsection, we investigate the ATPC strategy proposed in [5]. This strategy consists in finding the transmitted power from a linear algebraic equation between the RSSI and the transmitted power. The linearity of the algebraic equation is justified by a measurement campaign in which we study the relation between the transmission power and the RSSI. The whole experimental equipment was lent to us by the Wytek company which also participated in the implementation of programs. Our indoor experiments, in a classroom in the university of Poitiers, ensure the line-of-sight propagation between the CS and the WS. The experimental site is shown in the figure 1 .

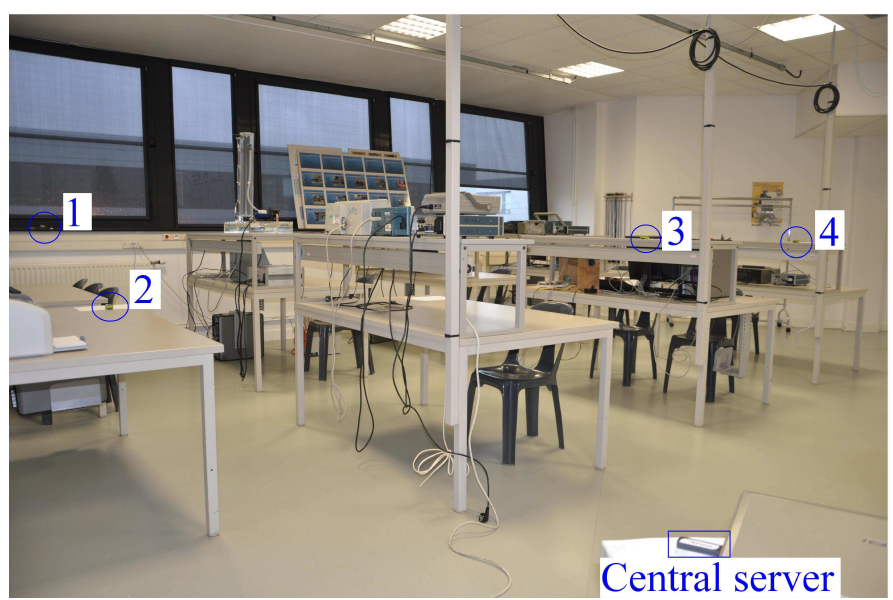

Figure 1: Experimental site

We have a CS and four fixed WS at different fixed positions numbered on the figure 1. We have alternatively measured the RSSI between the CS and the WS. With the ZigBee standard, each WS sends data in turn to the CS 
in order to avoid interferences [13]. The figure 2 shows the relations between the transmission power of each WS and the RSSI.
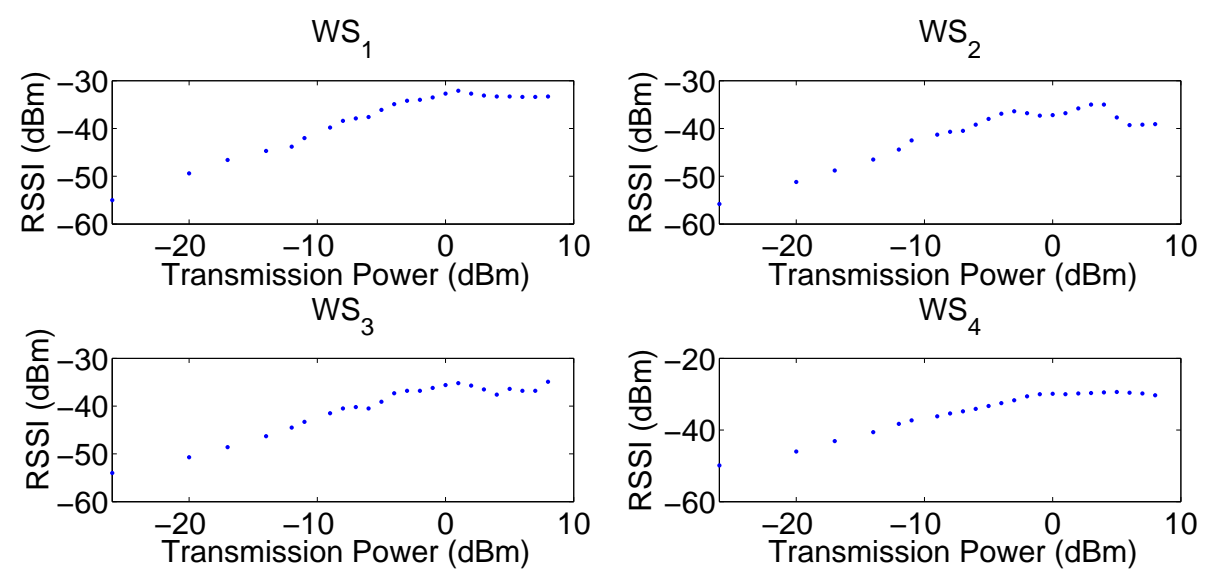

Figure 2: RSSI vs transmission power

These relations can also be found by using the propagation loss $L_{P_{T X} \rightarrow P_{R X}}$ of the free-space propagation model given by

$$
L_{P_{T X} \rightarrow P_{R X}}(d B)=P_{T X}-P_{R X}=32.45+20 \log (d)+20 \log (f)
$$

where $d$ is the distance (in $\mathrm{km}$ ) between the CS and the WS, $f$ the carrier frequency (in $\mathrm{MHz}$ ), $P_{R X}$ the received power and $P_{T X}$ the transmission power in $\mathrm{dB}$ [14]. The relation between the RSSI and the received power is given by

$$
\mathrm{RSSI}=10 \log \left(\frac{p_{R X}}{p_{R F}}\right)
$$

where $p_{R X}$ and $p_{R F}$ are respectively the received power and the reference power in the linear domain [15, Chapter 14]. The relationship between $p_{R X}$ 
and $P_{R X}$ is given by the following equation

$$
P_{R X}[d B m]=10 \log \left(p_{R X}\right) .
$$

In the case of the $\mathrm{WS}_{1}$ and according to the figure 2 , we deduce the experiment value $L_{P_{T X} \rightarrow \mathrm{RSSI}}(d B) \approx 28 \mathrm{~dB}$ which is the loss between $P_{T X}$ and the RSSI, i.e. RSSI $=-L_{P_{T X} \rightarrow \mathrm{RSSI}}+P_{T X}$. If we calculate this value from (1) and (2), we have

$$
\begin{aligned}
L_{P_{T X} \rightarrow \mathrm{RSSI}} & =P_{T X}-10 \log \left(\frac{p_{R X}}{p_{R F}}\right) \\
& =P_{T X}-10 \log \left(p_{R X}\right)+10 \log \left(p_{R F}\right) \\
& =P_{T X}-P_{R X}+10 \log \left(p_{R F}\right) \\
& =L_{P_{T X} \rightarrow P_{R X}}+10 \log \left(p_{R F}\right) \\
& =32.45+20 \log (d)+20 \log (f)+10 \log \left(p_{R F}\right)
\end{aligned}
$$

For $d=6 \mathrm{~m}, f=2.4 \mathrm{Ghz}$, and $p_{R F}=0.001 \mathrm{~W}$, we obtain the theoretical value $L_{P_{T X} \rightarrow R S S I} \approx 25.62 \mathrm{~dB}$ which is close to the experiment value.

We observe that the RSSI increases linearly for a low transmission power below $0 \mathrm{dBm}$ which is the domain of interest for the power control in this article. Therefore, we can define an approximate model as follows

$$
X_{i}(n)=a P_{i}(n)+b_{i}(n)+N_{i}(n)
$$


where $X_{i}$ is the RSSI, $P_{i}$ the transmission power, $N_{i}$ the noise, $n$ the transmission time, $a, b_{i}$ the parameters of the model and $i$ the considered $\mathrm{WS}_{i}$, $i=1,2,3,4$. The constant $a$ depends on the choice of the equipment and the standard but does not depend on the environment. The parameters $b_{i}$ depend on the position of the $\mathrm{WS}_{i}$ with respect to the CS. The additive term $N_{i}$ in the model (4) allows to take into account the imperfect linear model $X_{i}(n)=a P_{i}(n)+b_{i}(n)$. The parameters $a$ and $b_{i}$ of each $\mathrm{WS}_{i}$ are easily identified by using a least square estimation method from measurements given in the figure 2. The reader may notice that a change of environment for a $\mathrm{WS}_{i}$ implies a change of $b_{i}$ which leads to a new line parallel to the initial line in the figure 2 .

The initial values of the parameters $b_{i}$ are given in the table 1 . The value

\begin{tabular}{|c|c|}
\hline & $b_{i}$ \\
\hline $\mathrm{WS}_{1}$ & -33.59 \\
\hline $\mathrm{WS}_{2}$ & -36.11 \\
\hline $\mathrm{WS}_{3}$ & -35.73 \\
\hline $\mathrm{WS}_{4}$ & -29.13 \\
\hline
\end{tabular}

Table 1: Values of the parameters $b_{i}$ for the $\mathrm{WS}_{i}$

of $a$, which is the slope of the lines in the figure 2 , is approximately equal to 0.75 .

The other objective of the measurement campaign is to find the minimum value of the RSSI which ensures a robust link between the CS and a WS, named $\mathrm{RSSI}_{\text {min }}$. This value of $\mathrm{RSSI}_{\text {min }}$ does not depend on the environment but only on the equipment and the standard. We initialize the transmission 
power $\mathrm{P}_{T X}$ at $\mathrm{P}_{\max }$, if the reception is confirmed by a number of consecutive acknowledgement, we decrease the $\mathrm{P}_{T X}$ of one power level. We continue to decrease the $\mathrm{P}_{T X}$ until the link is interrupted and we find a minimum value of $\mathrm{P}_{\min }$. If we have a number of consecutive no acknowledgement of data, we increase the transmission power $\mathrm{P}_{T X}$ to connect with the CS. We take the $\mathrm{RSSI}_{\text {min }}$ as the value of the RSSI at the minimum power, i.e. $\mathrm{RSSI}_{\text {min }}=$ $X_{i}\left(P_{\text {min }}\right)$ and it is the same for all $\mathrm{WS}_{i}$. We find that $\mathrm{RSSI}_{m i n}=-50 \mathrm{dBm}$.

The ATPC consists in finding the transmitted power from the knowledge of the values $a, b_{i}(n)$, and $\operatorname{RSSI}_{m i n}$ at the time $n$ by using the equation (4). The complete algorithm is given in [5]. A comparison of ATPC with the $\mathrm{MPC}$ and PFC strategies will be given in the section 4.

\section{The PFC strategy}

\subsection{The modeling}

In this subsection, we find a recurrence equation for the RSSI from the equation (4). The fact that the CS alternatively communicates with the WSs leads to a scalar discrete-time system. For WSNs, each WS adjusts its transmission power according to the information given by the CS. We have the following classical dynamical equation for the transmission power

$$
\begin{aligned}
& P_{i}(n+1)=P_{i}(n)+\Delta P_{i}(n) \\
& b_{i}(n+1)=b_{i}(n)+\Delta b_{i}(n)
\end{aligned}
$$


where $\Delta P_{i}(n)$ and $\Delta b_{i}(n)$ are respectively the update values of the transmission power and the parameter $b_{i}$ at the transmission time $n$ for the $\mathrm{WS}_{i}$. We also have

$$
N_{i}(n+1)=N_{i}(n)+\Delta N_{i}(n) .
$$

From (4), (5) and (6) we get

$$
X_{i}(n+1)=X_{i}(n)+a \Delta P_{i}(n)+\Delta b_{i}(n)+\Delta N_{i}(n)
$$

which leads to

$$
X_{i}(n+1)=X_{i}(n)+U_{i}(n)+D_{i}(n)
$$

where $U_{i}(n)=a \Delta P_{i}(n)$ is the control variable and $D_{i}(n)=\Delta b_{i}(n)+\Delta N_{i}(n)$ the disturbance. We suppose that $D_{i}(n)$ is bounded by $\epsilon_{i}$. This bound $\epsilon_{i}$ depends on the environment and the transmission time of the system (7).

\subsection{The controller}

The basic definitions on asymptotic stability and Lyapunov functions for discrete-time systems with disturbances of the form

$$
x(n+1)=f(n, x(n), d(n)), \quad n \in \mathbb{N}
$$

where the state variable $x(.) \in \mathbb{R}^{n}$, the disturbances $d(.) \in \Omega$ with $\Omega \subset \mathbb{R}^{m}$, and $f$ is a continuous function, are given in [16, Definitions 2.1 and 2.6]. The Lyapunov theorems for discrete-time systems used in this article are 
given in $[17,18]$ without disturbances and in [16, Theorem 1] and [19] with disturbances.

In this section, we provide a new simple feedback control of the form

$$
u(n)=k_{1} x(n)+\frac{k_{2}}{x(n)}, \quad k_{2}>0 .
$$

where a potential term $\frac{k_{2}}{x(n)}$ is added to a linear one $k_{1} x(n)$ in order to ensure the constraint on the state variable $x(n)$. It is an original approach first developed in [10] where the stabilization is achieved with $-\frac{1}{2}<k_{1}<0$ for perturbed systems. In the Theorem 1, we extend the validity of the PFC to the limit case $k_{1}=-\frac{1}{2}$ for nominal systems.

Theorem 1. Consider the scalar system given by

$$
x(n+1)=x(n)+u(n), \quad n \in \mathbb{N}
$$

where $x(n) \in \mathbb{R}$ is the state and $u(n) \in \mathbb{R}$ the input. Under the initial condition $x(0)>0$, the system (9) with the feedback control

$$
u(n)=-\frac{1}{2} x(n)+\frac{k_{2}}{x(n)}, \quad k_{2}>0
$$

is asymptotically stable and converges toward $\sqrt{2 k_{2}}$. Moreover, we have $x(n) \geq \sqrt{2 k_{2}}$ for all $n \in \mathbb{N}^{*}$.

Proof. With the following input $u(n)=k_{1} x(n)+\frac{k_{2}}{x(n)}$, we have $x(n+1)=$ $x(n)+k_{1} x(n)+\frac{k_{2}}{x(n)}$. An equilibrium point $\bar{x}$ is solution of the equation

$$
\bar{x}=\bar{x}+k_{1} \bar{x}+\frac{k_{2}}{\bar{x}}
$$


If $\bar{x} \neq 0$ then it leads to $\bar{x}^{2}=-\frac{k_{2}}{k_{1}}$. If $k_{1}<0$ and $k_{2}>0$ then we have a positive root $x^{+}=\sqrt{-\frac{k_{2}}{k_{1}}}$. Suppose in the following that $k_{1}<0$ and $k_{2}>0$.

First of all, it is easy to prove by induction that if $-1<k_{1}<0$ then $x(n)>0$ for all $n \in \mathbb{N}$. In the remainder of the proof, we consider $-1<$ $k_{1}<0$. Let us prove that for all $n \in \mathbb{N}^{*}, x(n) \geq x^{+}$. We seek the minimum value of the function $x(n+1)$ with respect to the variable $x(n)$, then we will impose that the minimum value is equal to $x^{+}$. For this, we calculate the derivative of $x(n+1)$ :

$$
\frac{\partial x(n+1)}{\partial x(n)}=\frac{\left(1+k_{1}\right) x(n)^{2}-k_{2}}{x(n)^{2}}
$$

for $x(n)>0$ and $n \in \mathbb{N}$. The equality $\frac{\partial x(n+1)}{\partial x(n)}=0$ is equivalent to $x(n)=$ $\pm \sqrt{\frac{k_{2}}{1+k_{1}}}$. By studying the sign of the derivative, we obtain $\min _{x(n)>0} x(n+$ $1)=2 \sqrt{k_{2}\left(1+k_{1}\right)}$. If this minimum value is equal to $x^{+}$then $x(n+1) \geq x^{+}$ for all $n \in \mathbb{N}^{*}$. It leads to

$$
2 \sqrt{k_{2}\left(1+k_{1}\right)}=\sqrt{-\frac{k_{2}}{k_{1}}}
$$

We deduce that $k_{1}=-\frac{1}{2}$. It is worth noting that it is not possible to have $2 \sqrt{k_{2}\left(1+k_{1}\right)}>\sqrt{-\frac{k_{2}}{k_{1}}}$ with $-1<k_{1}<0$ and $k_{2}>0$. We remark that although if $x(0)<x^{+}$, the value of $x(n)$ for all $n \in \mathbb{N}^{*}$ is always greater than $x^{+}$

Consider the following Lyapunov function $V(x(n))=x(n)-x^{+}$for $n \in$ 
$\mathbb{N}^{*}$. We have

$$
\begin{aligned}
\Delta V(x(n)) & =V(x(n+1))-V(x(n)) \\
& =\frac{-\frac{1}{2} x(n)^{2}+k_{2}}{x(n)} .
\end{aligned}
$$

It is easy to see that $\Delta V(x(n))<0$ if $x(n)>x^{+}=\sqrt{2 k_{2}}$. Therefore, we have $\Delta V(x(n))<0$ for all $x(n) \neq \sqrt{2 k_{2}}$ and all $n \in \mathbb{N}^{*}$. By using the Lyapunov theorem given in [17] and [18, Theorem 4.20], we deduce that the system (9) is asymptotically stable and converges toward $\sqrt{2 k_{2}}$.

The Theorem 2 extend the validity of the Theorem 1 for systems with disturbances.

Theorem 2. Consider the scalar system given by

$$
x(n+1)=x(n)+u(n)+d(n), \quad n \in \mathbb{N}
$$

where $x(n) \in \mathbb{R}$ is the state, $u(n) \in \mathbb{R}$ the input and $|d(n)| \leq \epsilon$ a bounded disturbance. Let $c \leq 0$, under the initial condition $x(0)>c$ the system (10) with the feedback control

$$
u(n)=-\frac{1}{2}(x(n)-c)+\frac{k_{2}}{x(n)-c}
$$

where $k_{2} \geq \frac{41+3 \sqrt{73}}{64} \epsilon^{2} \approx 1.0411 \epsilon^{2}$ is asymptotically stable with respect to the attractive interval $S=\left[-\epsilon+\sqrt{2 k_{2}}+c ; 3 \epsilon+\sqrt{9 \epsilon^{2}+2 k_{2}}+c\right]$. Moreover, we have $x(n) \geq-\epsilon+\sqrt{2 k_{2}}+c$ for all $n \in \mathbb{N}^{*}$.

Proof. We have two possible cases which depend on the sign of the initial condition $x(0)$ of the system (10).

First case: $x(0)>0$. We take $c=0$. Firstly, we seek the equilibrium points 
of the closed-loop system. With the following input $u(n)=-\frac{1}{2} x(n)+\frac{k_{2}}{x(n)}$, we have $x(n+1)=\frac{1}{2} x(n)+\frac{k_{2}}{x(n)}+d(n)$. The positive equilibrium interval is given by

$$
S_{e q}^{+}=\left\{\bar{x}>0: \bar{x}=\frac{1}{2} \cdot \bar{x}+\frac{k_{2}}{\bar{x}}+d(n) ;|d(n)| \leq \epsilon\right\}
$$

If $\bar{x} \neq 0$ then we have $\frac{1}{2} \bar{x}^{2}-d(n) \bar{x}-k_{2}=0$. If $k_{2}>0$ then we have a positive root $x^{+}(n)=d(n)+\sqrt{\Delta}$ where $\Delta=d(n)^{2}+2 k_{2}$. We obtain

$$
S_{e q}^{+}=\left[-\epsilon+\sqrt{\epsilon^{2}+2 k_{2}} ; \epsilon+\sqrt{\epsilon^{2}+2 k_{2}}\right] \text {. }
$$

We seek the minimum value of $x(n+1)$. For this, we calculate the derivative of $x(n+1)$ and we obtain

$$
\frac{\partial x(n+1)}{\partial x(n)}=\frac{\frac{1}{2} x(n)^{2}-k_{2}}{x(n)^{2}}
$$

for $x(n)>0$ and $n \in \mathbb{N}$. We have $\frac{\partial x(n+1)}{\partial x(n)}=0$ is equivalent to $x(n)= \pm \sqrt{2 k_{2}}$. So, the interval of minimum values of $x(n+1)$ is given by

$$
S_{\text {min }}=\left[\sqrt{2 k_{2}}-\epsilon ; \sqrt{2 k_{2}}+\epsilon\right]
$$

The smallest value of $S_{\min }$ is $\min S_{\min }=\sqrt{2 k_{2}}-\epsilon$. We want that $\min S_{\min }>$ 0 , therefore $k_{2}>\frac{\epsilon^{2}}{2}$ in the following. Thus, we have $x(n)>\min S_{\min }=$ $\sqrt{2 k_{2}}-\epsilon>0$ for all $n \in \mathbb{N}^{*}$. As $\min S_{\text {min }}$ is slightly smaller than $\min S_{e q}^{+}$, the right limit of the attractive interval is given by $\min S_{m i n}$. At this stage, 
the attractive interval is given by

$$
S_{0}=\left[-\epsilon+\sqrt{2 k_{2}} ; \epsilon+\sqrt{\epsilon^{2}+2 k_{2}}\right] \text {. }
$$

Now, we have to prove the asymptotic stability of system (10). Let us consider the following Lyapunov function $V(n)=x(n)-x^{+}(n)$ for $x(n)>$ $\epsilon+\sqrt{\epsilon^{2}+2 k_{2}}$ and $n \in \mathbb{N}^{*}$. The function $\Delta V(n, x(n))=V(x(n+1))-V(x(n))$ is given by

$$
\Delta V(n, x(n))=\frac{-\frac{1}{2} x(n)^{2}+\left(d(n)-x^{+}(n+1)+x^{+}(n)\right) x(n)+k_{2}}{x(n)} .
$$

It is easy to see that $\Delta V(n, x(n))<0$ if $x(n)>x^{*}(n)$ where

$$
\begin{aligned}
x^{*}(n) & =\left(d(n)-x^{+}(n+1)+x^{+}(n)\right) \\
& +\sqrt{\left(d(n)-x^{+}(n+1)+x^{+}(n)\right)^{2}+2 k_{2}} .
\end{aligned}
$$

The value $\max _{n \in \mathbb{N}^{*}} x^{*}(n)$ is equal to $3 \epsilon+\sqrt{9 \epsilon^{2}+2 k_{2}}$ for $d(n)=\epsilon, x^{+}(n)=$ $\epsilon+\sqrt{\epsilon^{2}+2 k_{2}}$, and $x^{+}(n+1)=-\epsilon+\sqrt{\epsilon^{2}+2 k_{2}}$. As the maximum value $\max _{n \in \mathbb{N}^{*}} x^{*}(n)$ is greater than $\max S_{e q}^{+}$, we define the attractive interval by

$$
S_{0}=\left[-\epsilon+\sqrt{2 k_{2}} ; 3 \epsilon+\sqrt{9 \epsilon^{2}+2 k_{2}}\right]
$$

and we have $\Delta V(n, x(n))<0$ for all $x(n)>3 \epsilon+\sqrt{9 \epsilon^{2}+2 k_{2}}$.

To conclude, we have to prove that $S_{0}$ is invariant. Due to the previous 
calculus of $S_{\text {min }}$, we have $\min _{x(n) \in S_{0}} x(n+1)=\min S_{0}$. Now, let us calculate $\max _{x(n) \in S_{0}} x(n+1)$ and impose that $\max _{x(n) \in S_{0}} x(n+1) \leq \max S_{0}$. As the continuous function $x(n+1)$ with respect to the variable $x(n)$ is decreasing for $x(n) \in S_{1}=\left[-\epsilon+\sqrt{2 k_{2}} ; \sqrt{2 k_{2}}\right]$ and increasing for $x(n) \in S_{2}=\left[\sqrt{2 k_{2}} ; 3 \epsilon+\right.$ $\left.\sqrt{9 \epsilon^{2}+2 k_{2}}\right]$, where $S_{0}=S_{1} \cup S_{2}$, we only calculate the values of $x(n+1)$ at $\min S_{0}$ and at $\max S_{0}$ due to the intermediate value theorem. At the value $x(n)=\min S_{0}$, we have

$$
\left.x(n+1)\right|_{\min S_{0}}=\frac{\sqrt{2 k_{2}}-\epsilon}{2}+\frac{k_{2}}{\sqrt{2 k_{2}}-\epsilon} \leq \frac{\sqrt{2 k_{2}}+\epsilon}{2}+\frac{k_{2}}{\sqrt{2 k_{2}}-\epsilon}=\frac{\left(\sqrt{2 k_{2}}+\epsilon\right)\left(4 k_{2}-\epsilon^{2}\right)}{2\left(2 k_{2}-\epsilon^{2}\right)} .
$$

Moreover, we have

$$
\begin{aligned}
\max S_{0} & =3 \epsilon+\sqrt{9 \epsilon^{2}+2 k_{2}} \\
& =\frac{\left(4 k_{2}-\epsilon^{2}\right)\left(\epsilon+\sqrt{9 \epsilon^{2}+2 k_{2}}\right)}{2\left(2 k_{2}-\epsilon^{2}\right)} \\
& +\frac{2 \epsilon\left(4 k_{2}-\epsilon^{2}\right)-\epsilon^{2}\left(3 \epsilon+\sqrt{9 \epsilon^{2}+2 k_{2}}\right)}{2\left(2 k_{2}-\epsilon^{2}\right)}
\end{aligned}
$$

We remark that if

$$
\frac{2 \epsilon\left(4 k_{2}-\epsilon^{2}\right)-\epsilon^{2}\left(3 \epsilon+\sqrt{9 \epsilon^{2}+2 k_{2}}\right)}{2\left(2 k_{2}-\epsilon^{2}\right)} \geq 0
$$

then $\left.x(n+1)\right|_{\min S} \leq \max S_{0}$. It leads to the condition

$$
2\left(4 k_{2}-\epsilon^{2}\right)-\epsilon\left(3 \epsilon+\sqrt{9 \epsilon^{2}+2 k_{2}}\right) \geq 0
$$


which is equivalent to

$$
32 k_{2}^{2}-41 \epsilon^{2} k_{2}+8 \epsilon^{4} \geq 0
$$

It is easy to see that for $k_{2} \geq \frac{41+3 \sqrt{73}}{64} \epsilon^{2} \approx 1.0411 \epsilon^{2}$ the inequality (12) is satisfied. At the value $x(n)=\max S_{0}$, we have $\left.x(n+1)\right|_{\max S_{0}}<\max S_{0}$ and the desired condition is checked. Therefore, the attractive interval $S_{0}$ is invariant if $k_{2} \geq \frac{41+3 \sqrt{73}}{64} \epsilon^{2} \approx 1.0411 \epsilon^{2}$.

By using the Lyapunov theorem for discrete-time systems given in $[16$, Theorem 1], we conlude that if $k_{2} \geq \frac{41+3 \sqrt{73}}{64} \epsilon^{2}$ then the system (10) is asymptotically stable with respect to the attractive interval $S_{0}$.

Second case: $x(0) \leq 0$. From equations (10) and (11) we have

$$
x(n+1)=x(n)+k_{1}(x(n)-c)+\frac{k_{2}}{x(n)-c}+d(n) .
$$

Subtracting $c$ in both sides of the equation (13), we get

$$
x(n+1)-c=x(n)-c+k_{1}(x(n)-c)+\frac{k_{2}}{x(n)-c}+d(n) .
$$

Consider the following change of variable

$$
\psi(n)=x(n)-c
$$


that leads to the new system

$$
\psi(n+1)=\left(k_{1}+1\right) \psi(n)+\frac{k_{2}}{\psi(n)}+d(n)
$$

We take $c<x(0)$ and it leads to $\psi(0)>0$. Therefore, we can apply the proof

of the first case. We deduce that if $c<x(0)$, and $k_{2} \geq \frac{41+3 \sqrt{73}}{64} \epsilon^{2} \approx 1.0411 \epsilon^{2}$ then the system (10) is asymptotically stable with respect to the attractive interval $S$

$$
S=\left[-\epsilon+\sqrt{2 k_{2}}+c ; 3 \epsilon+\sqrt{9 \epsilon^{2}+2 k_{2}}+c\right]
$$

\section{Experimental results}

\subsection{Power control}

We apply the PFC (11) to each $\mathrm{WS}_{i}$ under the static environment condition which implies that $\Delta b_{i}=0$. We take a transmission time of $5 \mathrm{~s}$ in our application. The threshold value of the RSSI is given in the section 2 by $\mathrm{RSSI}_{\text {min }}=-50 \mathrm{dBm}$. We suppose that $\epsilon_{i}$ is the maximum value of the disturbances $D_{i}(n)$. We chose $\epsilon_{i}=5 \mathrm{dBm}$ as the security margin which can take into account the variations of RSSI due to the presence of a person between the CS and the WS [20]. According to the Theorem 2, we now search for the values of $c$ and $k_{2}$. We take $c=-51 \mathrm{dBm}$ to satisfy $c<\mathrm{RSSI}_{\text {min }}$ for all $\mathrm{WS}_{i}$. In order to have $X_{i}(n) \geq \operatorname{RSSI}_{\min }$ where $X_{i}$ is given by the equation (7), we 
impose $-\epsilon+\sqrt{2 k_{2}}+c \geq \operatorname{RSSI}_{\text {min }}$. It leads to $k_{2} \geq \frac{\left(\operatorname{RSSI}_{\min }+\epsilon-c\right)^{2}}{2}$. We can take the value of $k_{2}=20$ for all $\mathrm{WS}_{i}$.

First, we initialize the transmission power at the maximum value which is $8 \mathrm{dBm}$. The figures 3, 4, 5, and 6 illustrate the evolution of the transmission power and the RSSI in function of the transmission time for each WS by using the PFC strategy, the ATPC strategy and the MPC strategy having a prediction and control horizon of 1 . We note that after few transmission times, the transmission power decreases and then stays quasi constant. Moreover, the RSSI is always higher than the $\mathrm{RSSI}_{\text {min }}$ which is equal to $-50 \mathrm{dBm}$. The final RSSI value of all $\mathrm{WS}_{i}$ converges towards around $-45 \mathrm{dBm}$ because a security margin of $5 \mathrm{dBm}$ has been taken into account. We also observe that the efficiency of all strategies is quite similar in the steady state. For the ATPC approach, the RSSI converges towards the optimal value in one transmission time due to the knowledge of $b(n)$.
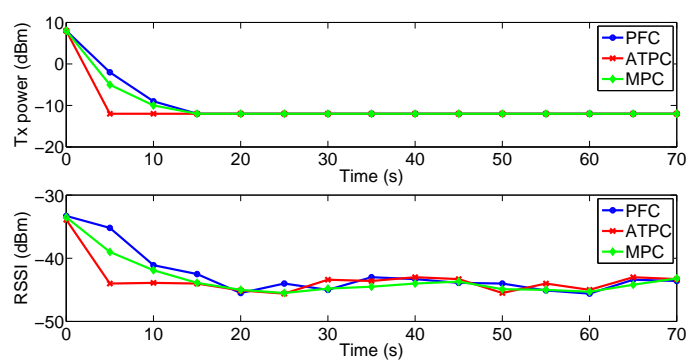

Figure 3: Evolution of the transmission power and the RSSI for the $\mathrm{WS}_{1}$ with the initial condition of $8 \mathrm{dBm}$ 

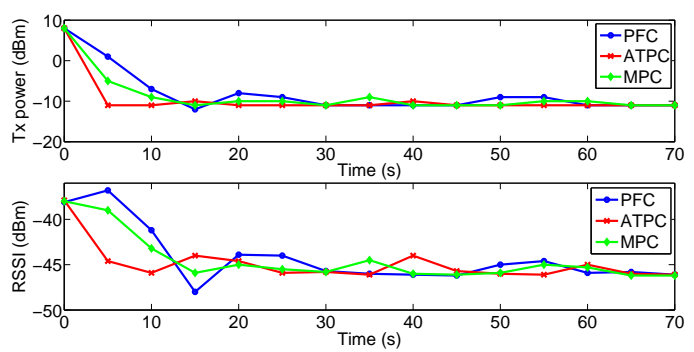

Figure 4: Evolution of the transmission power and the RSSI for the $\mathrm{WS}_{2}$ with the initial condition of $8 \mathrm{dBm}$
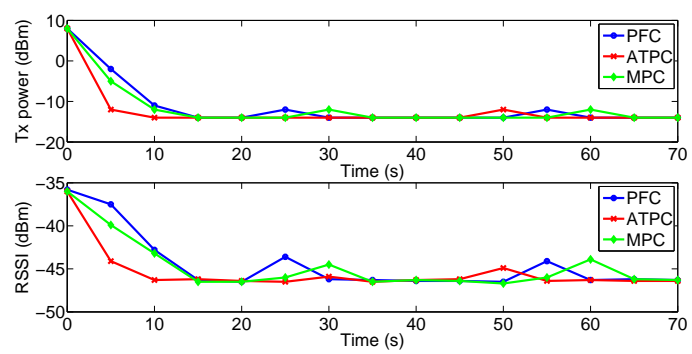

Figure 5: Evolution of the transmission power and the RSSI for the $\mathrm{WS}_{3}$ with the initial condition of $8 \mathrm{dBm}$
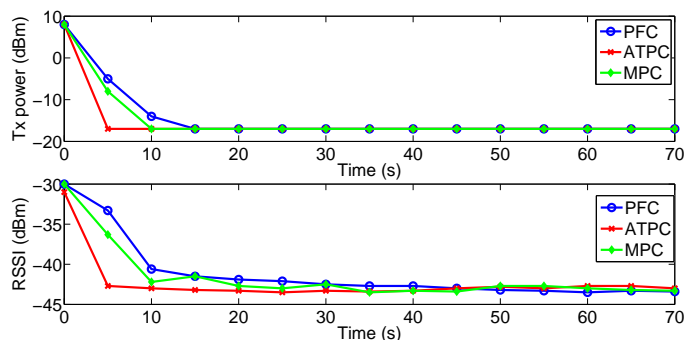

Figure 6: Evolution of the transmission power and the RSSI for the $\mathrm{WS}_{4}$ with the initial condition of $8 \mathrm{dBm}$

However, we see in the figure 7 that the execution time of the $\mathrm{PFC}$ is shorter than the one of the MPC and ATPC. This is due to the fact that we have no prediction and no estimation of $b(n)$ to do with the PFC. The 
PFC leads to a better energy efficiency than the MPC and ATPC at the level of the CS and this is interesting when the number of WSs becomes high or when the CS has also a limited energy.

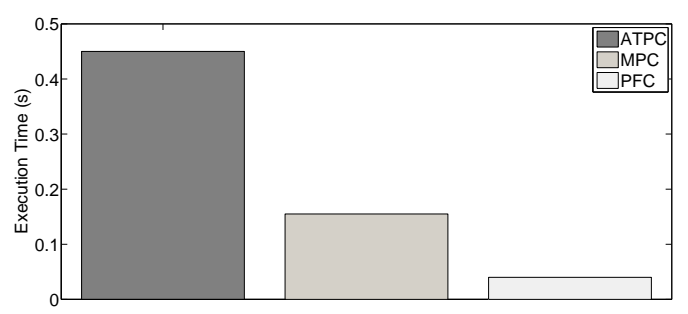

Figure 7: Execution time of controllers for a time period of 1 day

Now, we will see that the PFC strategy is able to adapt to the change of the environment. It can take into account only the slow fading which affects the system for a long time. For instance, the power control is not able to take into account a fading due to a person passing between the CS and the WS due to the transmission time of $5 \mathrm{~s}$ and this is the reason why we take a security margin of $5 \mathrm{dBm}$. However, it can adapt to a modification of the environment over a long time due for instance to an obstacle placed between the CS and the WS. In our real application, we put an obstacle between the CS and the $\mathrm{WS}_{4}$ in the time between two data transmissions and we observe a drop of $-10 \mathrm{dBm}$ of the RSSI with respect to the initial environment. The figure 8 shows the variation of the RSSI and the adaptation of the power control. We observe that the power increases to its maximum value of $8 \mathrm{dBm}$ after a significant drop of the RSSI. Then, it converges towards its final value of $-6 \mathrm{dBm}$ which ensures that the RSSI is always higher than the $\mathrm{RSSI}_{\text {min }}$ 
in the new environment.
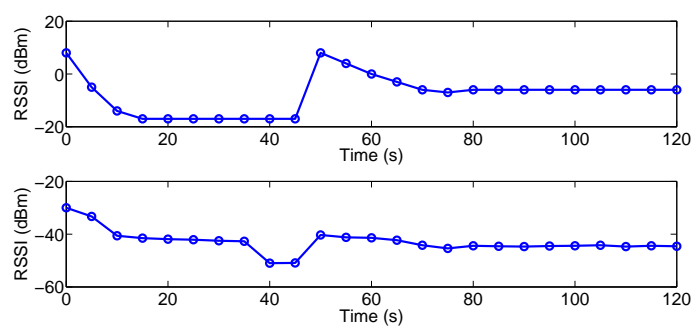

Figure 8: Evolution of the transmission power and the RSSI for the $\mathrm{WS}_{4}$ with the obstacle

\subsection{Energy consumption}

The results show the validity of our approach. Initially, the WSs were configured to transmit at the maximum power of $8 \mathrm{dBm}$. The implementation of our PFC has allowed to decrease the transmitted power of each $\mathrm{WS}_{i}$ as shown in the table 2 .

\begin{tabular}{|c|c|}
\hline & $P_{i \min }(\mathrm{dBm})$ \\
\hline $\mathrm{WS}_{1}$ & -12 \\
\hline $\mathrm{WS}_{2}$ & -9 \\
\hline $\mathrm{WS}_{3}$ & -12 \\
\hline $\mathrm{WS}_{4}$ & -17 \\
\hline
\end{tabular}

Table 2: Transmission power for each $\mathrm{WS}_{i}$

In the ZigBee standard, the energy consumption of a WS is composed of five mains parts: the wake up of the WS, the listening to the channel, the Carrier Sense Multiple Access-Collision Avoidance (CSMA-CA) protocol, the transmission of data (TX) and the acknowledgment of the Media Access Control (ACK-MAC) layer. After the measure campaign, we observe that the ratio between the transmission power and the total power varies due to 
the channel condition and the data sent. It is illustrated in the figure 9. We can only reduce the power of the TX part by using a power control.

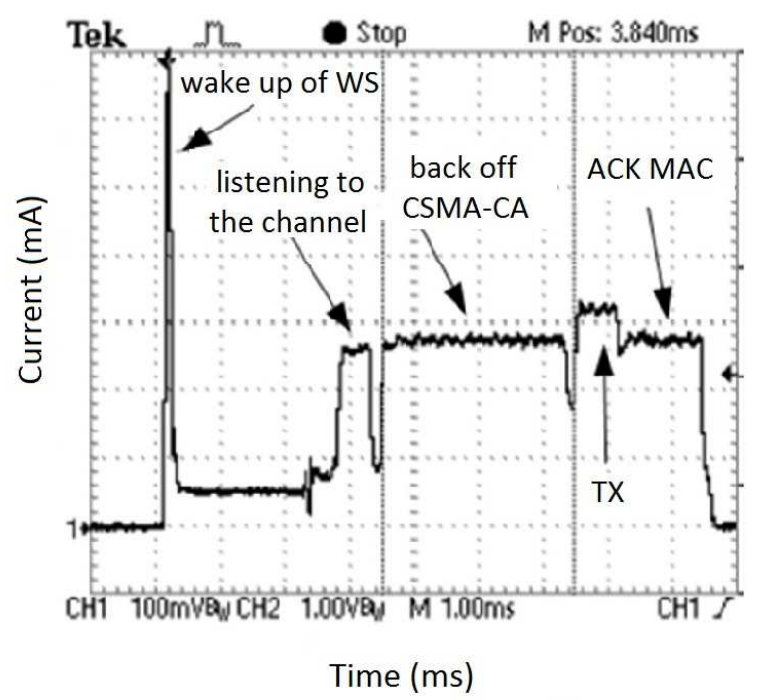

Figure 9: Energy consumption of a WS

Now, we are interested in the energy saving caused by the power control. For this, we make an energy saving test. We set the transmission power $\mathrm{P}_{T X}$ of the $\mathrm{WS}_{1}, \mathrm{WS}_{2}, \mathrm{WS}_{3}, \mathrm{WS}_{4}$ at $8 \mathrm{dBm},-9 \mathrm{dBm},-12 \mathrm{dBm},-17 \mathrm{dBm}$ respectively. The power of the $\mathrm{WS}_{1}$ is at the maximum and it was the reference for this test. The necessary time for each $\mathrm{WS}_{i}$ to fully discharge its battery is given in the table 3 . 


\begin{tabular}{|c|c|c|c|}
\hline & $\mathrm{P}_{T x}(\mathrm{dBm})$ & Time $T_{i}($ day $)$ & $G_{i}(\%)$ \\
\hline $\mathrm{WS}_{1}$ & 8 & 3.3682 & 0 \\
\hline $\mathrm{WS}_{2}$ & -9 & 3.5521 & 5.46 \\
\hline $\mathrm{WS}_{3}$ & -12 & 3.6424 & 8.14 \\
\hline $\mathrm{WS}_{4}$ & -17 & 3.6684 & 8.46 \\
\hline
\end{tabular}

Table 3: Energy saving with the PFC

We calculate the gain operating time $\mathrm{G}_{i}$ with respect to $\mathrm{WS}_{1}$ for each $\mathrm{WS}_{i}(i=2,3,4)$ by using the formula

$$
G_{i}=\frac{T_{i}-T_{1}}{T_{1}} \quad i=2,3,4
$$

where $T_{i}$ is the discharge time for the $\mathrm{WS}_{i}$. The gains $G_{i}$ are given in the table 3. Thus, we can extend the lifetime of the sensors of about $5 \%$ to $10 \%$.

\section{Conclusion}

In this article, we have developed a feedback control strategy for scalar discrete-time systems having a constraint on the state variable. This strategy is based on a nonlinear PFC. It is well suited for the power control of a WSN due to the constraint on the minimum value of the RSSI imposed by the telecommunications standards. This technique is applied to a real WSN of four WSs and is compared with the ATPC and MPC strategies. The PFC allows to reduce the transmission power and then the energy consumption of the WS device. Moreover, it has a better execution time than the ATPC and MPC strategies which leads to a lower energy consumption at the level 
of the CS.

\section{Acknowledgments}

The authors would like to thank Christian Chatellier, Yoann Hilairet and Patrick Pailler from the Wytek compagny for the loan of the equipment but also for their technical expertise.

[1] K. Sohraby, D. Minoli, T. Znati, Wireless sensor networks: technology, protocols, and applications, John Wiley \& Sons, 2007.

[2] A. Elahi, A. Gschwender, ZigBee wireless sensor and control network, Prentice Hall, 2009.

[3] T. He, S. Krishnamurthy, J. A. Stankovic, T. Abdelzaher, L. Luo, R. Stoleru, T. Yan, L. Gu, J. Hui, B. Krogh, Energy-efficient surveillance system using wireless sensor networks, in: International Conference on Mobile systems, applications, and services, Boston, USA, 2004, pp. 270283.

[4] G. Werner-Allen, K. Lorincz, M. Ruiz, O. Marcillo, J. Johnson, J. Lees, M. Welsh, Deploying a Wireless Sensor Network on an Active Volcano, IEEE Internet Computing 10 (2) (2006) 18-25.

[5] S. Lin, J. Zhang, G. Zhou, L. Gu, J. A. Stankovic, T. He, ATPC: adaptive transmission power control for wireless sensor networks, in: 
ACM International Conference on Embedded Networked Sensor Systems, Boulder, USA, 2006, pp. 223-236.

[6] E. Altman, Z. Altman, S-modular games and power control in wireless networks, IEEE Transactions on Automatic Control 48 (5) (2003) 839842.

[7] S. M. Mahdi Alavi, M. J. Walsh, M. J. Hayes, Robust distributed active power control technique for IEEE 802.15.4 wireless sensor networks a quantitative feedback theory approach, Control Engineering Practice 17 (7) (2009) 805-814.

[8] A. Bemporad, M. Morari, V. Dua, E. N. Pistikopoulos, The explicit linear quadratic regulator for constrained systems, Automatica 38 (1) (2002) 3-20.

[9] P. O. Gutman, A linear programming regulator applied to hydroelectric reservoir level control, Automatica 22 (5) (1986) 533-541.

[10] D. D. Phan, E. Moulay, P. Coirault, F. Launay, P. Combeau, Potential feedback control for the power control in LTE, IEEE Transactions on Automatic Controldoi:10.1109/TAC.2014.2382154.

[11] N. E. Leonard, E. Fiorelli, Virtual leaders, artificial potentials and coordinated control of groups, in: IEEE Conference on Decision and Control, Orlando, USA, 2001, pp. 2968-2973. 
[12] E. Rimon, D. E. Koditschek, Exact robot navigation using artificial potential functions, IEEE Transactions on Robotics and Automation 8 (5) (1992) 501-518.

[13] S. Farahani, ZigBee wireless networks and transceivers, Newnes, 2011.

[14] S. A. Ahson, M. Ilyas, WiMAX: applications, CRC press, 2007.

[15] S. J. Halder, J.-G. Park, W. Kim, Adaptive Filtering for Indoor Localization using ZigBee RSSI and LQI measurement, InTech, 2011.

[16] Z. P. Jiang, Y. Wang, A converse Lyapunov theorem for discrete-time systems with disturbances, Systems \& Control Letters 45 (1) (2002) $49-58$.

[17] K. Aström, B. Wittenmark, Computer-controlled systems: theory and design, Dover Publications, 2011.

[18] S. Elaydi, An introduction to difference equations, Springer, 2005.

[19] I. Karafyllis, Z. P. Jiang, Stability and stabilization of nonlinear systems, Communications and Control Engineering, Springer, 2011.

[20] D. Dessales, A.-M. Poussard, R. Vauzelle, N. Richard, Impact of people motion on radio link quality: Application to building monitoring WSN, in: IEEE International Symposium on Personal Indoor and Mobile Radio Communications, London, UK, 2013, pp. 2072-2076. 